\title{
Sperm swimming is more complicated than thought
}

In 1677, Antonie van Leeuwenhoek used one of his pioneering microscopes to observe sperm cells and first described their swimming as a lashing motion of their tails. This motion has been seen again and again and it became generally accepted that sperm wiggle in a symmetrical side-to-side motion. But this picture is wrong. Writing in Science Advances,

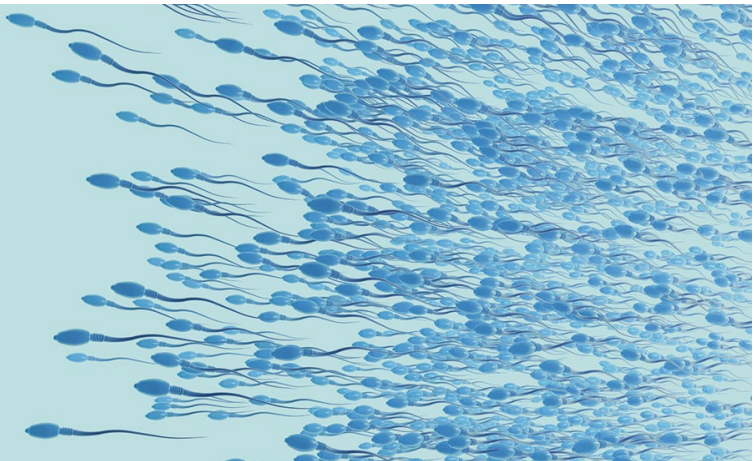

Hermes Gadêlha and colleagues report on high-frame-rate 3D video microscopy that reveals that sperm actually move with a combination of an asymmetrical travelling wave and a rolling motion. When combined and projected onto a $2 \mathrm{D}$ plane - as would be seen through a microscope like van Leeuwenhoek's - these motions look like a symmetrical wiggling.

Gadêlha et al. analysed their $3 \mathrm{D}$ recordings of sperm motion in two reference frames: one that moves with the sperm cell and another that co-moves and also rolls with the sperm about its swimming axis. This latter is the reference frame of the sperm itself. In the co-moving frame, the swimming motion is symmetrical when projected onto a plane, whereas in the co-moving rolling frame the projected motion is asymmetrical. In other words, the sperm's own wiggling is an asymmetrical motion, and it is the rotation that introduces symmetry. Such symmetry is vital, because the asymmetrical stroke alone would cause the sperm to swim around in circles, like a rowboat with only one oar.

That sperm swim with an asymmetrical stroke also answers a question that puzzles biologists. The microscopic structure of cross-section of the tail of a sperm cell is not symmetrical, and it is unclear how such an anisotropic structure would give rise to symmetrical back-and-forth motion. The results of Gadêlha et al. resolve this apparent paradox.

Zoe Budrikis

ORIGINAL ARTICLE Gadêlha, H. et al. Human sperm uses asymmetric and anisotropic flagellar controls to regulate swimming symmetry and cell steering. Sci. Adv. 6, eaba5168 (2020) 